Japan; ${ }^{2}$ Department of Epidemiology, University of Tsukuba, Tsukuba, Ibaraki, Japan; ${ }^{3}$ Social Services Division, Southern District Head Branch, Ibaraki Prefectural Government, Tsuchiura, Ibaraki, Japan; ${ }^{4}$ Graduate School of Comprehensive Human Sciences, Master's Program in Medical Sciences, University of Tsukuba, Tsukuba, Ibaraki, Japan; ${ }^{5}$ Health Services Disease Control Division, Department of Health and Social Services, Ibaraki Prefectural Government, Mito, Ibaraki, Japan

Introduction The $2009 \mathrm{~A} / \mathrm{H} 1 \mathrm{~N} 1$ influenza spread also in Japan. Many students were absent in elementary schools, To prevent its expansion, many school decided class closure with their original length under rough guideline by local education board. However, its effect had not been clear. The objective was to examine whether or not the class closure length related to the change of absentees.

Methods Subject was all the classes in elementary schools in T city that class closure was carried in the period from 1 September to 24 December in 2009. We sent the questionnaire including the questions (1) the number of students in class, (2) the number of absentees on the day, (3) whether or not class closure was carried out on the day, to the school principal, and asked school nurses to write under each class attendance book and to return them by post mail. The length of class closure and the change of absentees before and after class closure was analysed by $\chi^{2}$ test with statistical soft R2.11.1

Results 16 of total 37 elementary schools replied (43.2\%), and 103 classes of 15 schools with the closure were analysed. It revealed statistical associations between class closure length and the change of the proportions of absentee $(\mathrm{p}<0.001)$, and between class closure length and the proportions of class that absentee was decreased $(\mathrm{p}<0.001)$

Conclusion There were tendencies that the number of absentee decreased after class closure, and the longer class closure days, the fewer absentees changed.

\section{SP3-50 SERUM HEPATOCYTE GROWTH FACTOR LEVELS AND MORTALITIES FROM CANCER IN APPARENTLY HEALTHY GENERAL POPULATION}

doi:10.1136/jech.2011.1429760.50

\section{SP3-51 ACCESS TO TB PATIENTS OF DIRECTLY OBSERVED THERAPY (DOTS) DURING NATURAL DISASTERS IN BIHAR, INDIA}

doi:10.1136/jech.2011.1429760.51

R Kumar.* Integrated Disease Surveillance Project, Pauri, Uttarakhand, India

Introduction This pilot study attempts to look at pattern and problem of adherence to DOTS by TB patient during flooding in Bihar, India.

Methods A cross-sectional survey was undertaken among 21 (female $\mathrm{n}=9$ ) TB patients who were participating in DOTS and faced the problem of flooding in Muzaffarpur district of Bihar, India. They were interviewed to collect information on age, sex, education, occupation, duration of flooding in the village, discontinuity period due to flooding and other factors, change in DOT provider, and knowledge on importance of continuing treatment using a semistructured questionnaire.

Results Mean duration of flooding was 92.9 days (SD 32.4). Mean discontinuity period due to flooding was 26.9 days (SD 24.0). In the Fisher's Exact Test, Female TB treatment beneficiaries were found to more affected than males $(\mathrm{p}<0.005)$. Following discontinuing DOTS, $3(14.3 \%)$ participants thought that their disease would not be cured, 8 (38.1\%) participants thought their TB disease would come back, whereas and $9.5 \%$ said they did not know.

Conclusion Although flooding lead to discontinuity in treatment to many of the TB patients on DOTS. This may lead to antituberculosis drug resistance. The finding that females discontinued treatment more frequently than males needs to be highlighted. The TB control programme should look further in to this and take appropriate measures to address the issue.

\section{SP3-52 A DIFFERENCE IN ADRS (ADVERSE DRUG REACTIONS) MORTALITY RATE IN THAI TUBERCULOSIS PATIENTS BETWEEN YEAR 2008 AND 2009}

doi:10.1136/jech.2011.1429760.52

S Wechwithan, ${ }^{*}$ P Sriphiromya. Health product Vigilance Center, Nonthaburi, Thailand

Background Surveillance of adverse drug reactions in Thailand is conducted through the spontaneous voluntary reporting system by hospital pharmacists and healthcare professionals. Adverse drug reactions reports have been collected in national spontaneous reporting database called Thaivigibase since year 1985. Public health program using medicine in AIDS, Tuberculosis (TB) control program have collected the patients' records. Integrating public health program in TB patients and spontaneous reporting system can receive $\mathrm{ADR}$ mortality rate compare difference in anti-tuberculosis drug group. This ADR mortality rate may reflect $\mathrm{TB}$ drug group safety surveillance system.

Objective This study is aimed to compare difference in ADRs mortality rate in Thai tuberculosis patients, between year 2008 and 2009.

Study Design Descriptive observational study design is used for this study.

Materials and Methods Adverse reaction reports of patients to anti tuberculosis drugs from Thaivigibase and $\mathrm{TB}$ patient disease surveillance database from Bureau of Epidemiology during year 2008-2009 were retrieved and calculated. The pattern of spontaneous fatal adverse reactions to anti-tuberculosis drugs were described by analysing the data from Thaivigibase between year 2008 and 2009.

Results/Conclusion ADRs mortality rates to anti-tuberculosis drugs were 1.97 per 1000 patients in year 2009 compared with 4.35 per 1000 patients in year 2008. Stevens-Johnson Syndrome and 
hepatitis resulting in fatal outcome were detected in patients taking rifampicin, ethambutol, isoniazid and pyrazinamide. Anaphylactic shock was observed in patients with injected streptomycin. Skin and appendages disorders was the most common adverse effects reported. The result can be predictor of TB surveillance system to develop plan for TB patients surveillance.

\section{SP3-53 CHANGES IN THE NUMBER OF ORAL HEALTH-RELATED QUALITY OF LIFE IMPACTS IN ELDERLY PEOPLE}

doi:10.1136/jech.2011.1429760.53

F Andrade, ${ }^{*}$ M L Lebrão, J Santos, Y Duarte. University of São Paulo, São Paulo/São Paulo, Brazil

Objectives The aim of this study was to evaluate the pattern of changes in the number of oral-health-related-quality of life (OHRQoL) impacts and investigate its relationship with selfreported oral health measurements in a sample of communitydwelling elderly from São Paulo-Brazil.

Methods The sample consisted of 747, 65 and older people, enrolled in the Health, Well-being and Ageing cohort-study. OHRQoL negative-impacts were measured using the General Oral Health Index-GOHAI and calculated as the number of items reported as "always" or "often." Self-reported oral health measurements included: number of teeth lost and use of prosthesis. Changes scores were calculated by subtracting the number of impacts at baseline from the impacts at follow-up. A positive change score indicates an increment in the number of impacts, a negative score indicates a decrement and a change score of zero represents no change. Multinomial logistic regression analysis was used, estimating values for $\mathrm{OR}$ and a $95 \% \mathrm{CI}$. A design effect correction was made using the Stata survey command to analyse data coming from complex samples.

Results Most people had no change in the number of impacts and $21.80 \%$ had a decrement. Increment was related with tooth loss $\geq 16$ (OR 1.74), use of dental prosthesis (OR 0.54), schooling $\leq 3$ years (OR 1.65) and age (OR 1.03). Decrement was associated with tooth loss $\geq 16$ (OR 2.61), use of dental prosthesis (OR 0.52).

Conclusion Changes in OHRQoL impacts were significantly related with the number of teeth lost and use of prosthesis even after controlling for socioeconomic factors.

\section{SP3-54 MIDTERM CONSEQUENCES ON HEALTH OF THE EARTHOUAKE OF 6 APRIL 2009 IN L'AQUILA (ITALY), ASSESSED BY THE BEHAVIOURAL RISK FACTOR SURVEILLANCE SYSTEM PASSI}

doi:10.1136/jech.2011.1429760.54 disorder (PTSD) with the objective to establish a knowledge base for better informed public health decisions.

Methods From June to October 2010, 958 people living in earthquake area were interviewed by phone (response rate 95\%), using the properly modified PASSI questionnaire.

Results $92 \%$ of interviewees were in the earthquake area at time of event; $5 \%$ were injured; $10 \%$ knew an injured person, $40 \%$ knew a person killed by the earthquake among their relatives or friends, $35 \%$ had to leave their home due to severe damage, $45 \%$ reported economic losses and 15\% lost their job. $22 \%$ were displaced in a temporary public accommodation. $4 \%$ met the definition of PTSD and $16 \%$ of depression, higher for women and 50-69 aged years old. Conclusion The study provides an estimate of non-traumatic consequences of the earthquake, otherwise neglected but financially covered by the NHS, and is a useful tool for public health needs assessment and planning in case of a disaster.

\section{SP3-55 LAYERS OF COMPLEXITY IN INTERPRETING EFFECTIVENESS EVIDENCE}

doi:10.1136/jech.2011.1429760.55

${ }^{1}$ A Tannahill, ${ }^{*}$ M P Kelly. ${ }^{1}$ NHS Health Scotland, Glasgow, UK; ${ }^{2}$ National Institute for Health and Clinical Excellence, London, UK

Introduction There has been growing attention to using effectiveness evidence to guide public health and health improvement policies, strategies, programmes, and actions "on the ground." However, there has only been partial recognition of complications that have a material bearing on how such evidence is interpreted and translated into action. This paper aims to shed further light on such complications, capitalising on the authors' previous roles as "evidence originators" in academia and their more recently gained perspectives as "evidence interpreters" in national agencies.

Methods A particular randomised controlled trial in which the authors were directly involved-based on a workplace cardiovascular disease prevention programme-was revisited and used as an illustrative case study to elucidate important considerations in assessing and applying effectiveness evidence more generally.

Results Relatively obvious, and less obvious, complicating factors were identified in relation to defining the intervention, judging effectiveness, and transferability of findings. In addition, some "bigger picture" considerations were described, with individual interventions viewed as pieces in the health improvement "jigsaw" or as frames in the "movie" of ever-changing influences on population health.

Conclusion The layers of complexity uncovered in this work should be taken into account in designing, executing and reporting primary evaluative studies and reviews, in formulating recommendations for action, and in developing more fully fit-for-purpose approaches to evidence-informed public health and health improvement.

\section{SP3-56 TRENDS IN CARDIOVASCULAR RISK FACTORS IN THE UK, 1961-2011}

doi:10.1136/jech.2011.1429760.56 of L'Aquila, L'Aquila, Italy; ${ }^{3}$ Zadig, Roma, Italy; ${ }^{4}$ AUSL Pescara, Pescara, Italy; ${ }^{6}$ AUSL Teramo, Teramo, Italy; ${ }^{5}$ AUSL L'Aquila, Avezzano e Sulmona, L'Aquila, Italy; ${ }^{7}$ AUSL Lanciano-Vasto e Chieti, Chieti, Italy; ${ }^{8}$ AUSL Roma C, Roma, Italy

Intoduction 1 year after the earthquake in L'Aquila, all Abruzzo Local Health Units (LHU) with the University of L'Aquila, the Italian institute for health and the support of the Ministry of health, carried out a survey on midterm consequences of the earthquake on health. The analysis, based on the behavioural risk factors surveillance system PASSI, was aimed at assessing the prevalence of symptoms of depression and anxiety in adults, at monitoring health related behaviour and the prevalence of post-traumatic stress
Introduction Cardiovascular disease rates in the UK have been steadily declining over the past 50 years, mainly due to improvements in risk factors. This paper focuses on the behavioural risk factors and aims to document trends from 1961 to the present.

Methods We searched the peer-reviewed and grey literature from comparable estimates of prevalence of smoking, poor diet, alcohol consumption, physical inactivity and overweight and obesity, within the UK between 1961 and 2011. 